\title{
A Respiratory Therapist-Driven Asthma Pathway Reduced Hospital Length of Stay in the Pediatric Intensive Care Unit
}

\author{
Andrew G Miller, Kaitlyn E Haynes, Rachel M Gates, Kanecia O Zimmerman, Travis S Heath, \\ Kathleen W Bartlett, Heather S McLean, and Kyle J Rehder
}

\begin{abstract}
BACKGROUND: Asthma is a common reason for admissions to the pediatric intensive care unit (PICU). Since June 2014, our institution has used a pediatric asthma clinical pathway for all patients, including those in PICU. The pathway promotes respiratory therapist-driven bronchodilator weaning based on the Modified Pulmonary Index Score (MPIS). This pathway was associated with decreased hospital length of stay (LOS) for all pediatric asthma patients; however, the effect on PICU patients was unclear. We hypothesized that the implementation of a pediatric asthma pathway would reduce hospital LOS for asthmatic patients admitted to the PICU. METHODS: We retrospectively reviewed the medical records of all pediatric asthma subjects 2-17 y old admitted to our PICU before and after pathway initiation. Primary outcome was hospital LOS. Secondary outcomes were PICU LOS and time on continuous albuterol. Data were analyzed using the chi-square test for categorical data, the $t$ test for normally distributed data, and the Mann-Whitney test for nonparametric data. RESULTS: A total of 203 eligible subjects $(49$ in the pre-pathway group, 154 in the post group) were enrolled. There were no differences between groups for age, weight, gender, home medications, cause of exacerbation, medical history, or route of admission. There were significant decreases in median (interquartile range) hospital LOS (4.4 [2.9-6.6] d vs 2.7 [1.6-4.0] d, $P<.001)$, median PICU LOS (2.1 [1.3-4.0] d vs 1.6 [0.8-2.4] d, $P=.003)$, and median time on continuous albuterol (39 [25-85] h vs $27[13-42] \mathrm{h}, P=.001)$. Significantly more subjects in the post-pathway group were placed on high-flow nasal cannula (32\% vs $6 \%, P=.001)$ or noninvasive ventilation $(10 \%$ vs $4 \%, P=.02)$. CONCLUSION: The implementation of an asthma pathway was associated with decreased hospital LOS, PICU LOS, and time on continuous albuterol. There was also an increase in the use of high-flow nasal cannula and noninvasive ventilation after the implementation of this clinical pathway. Key words: asthma; status asthmaticus; respiratory therapy; asthma pathway; protocol; quality improvement; high-flow nasal cannula; propensity score matching. [Respir Care 2019;64(11):1325-1332. (C) 2019 Daedalus Enterprises]
\end{abstract}

\section{Introduction}

Asthma is one of the most common pediatric diseases, with up to $20 \%$ of children with asthma requiring visits to

\footnotetext{
Mr Miller, Ms Haynes, and Ms Gates are affiliated with Respiratory Care Services at Duke University Medical Center, Durham, North Carolina. Drs Zimmerman and Rehder are affiliated with the Division of Pediatric Critical Care Medicine, Duke University Medical Center, Durham, North Carolina. Dr Heath is affiliated with the Department of Pharmacy, Duke University Medical Center, Durham, North Carolina. Drs Bartlett and McLean are affiliated with the Division of Pediatric Hospital Medicine, Duke University Medical Center, Duke University Medical Center, Durham, North Carolina.
}

The authors have disclosed no conflicts of interest. the emergency department each year. ${ }^{1,2}$ Approximately 13 $50 \%$ of these children require hospital admission each year. ${ }^{3}$ Status asthmaticus is a severe, life-threatening asthma exacerbation that is refractory to short-acting bronchodila-

\footnotetext{
Ms Haynes presented a version of this paper as an Editors' Choice abstract at the AARC Congress, held December 4-7, 2018, in Las Vegas, Nevada.

Correspondence: Andrew G Miller RRT RRT-ACCS RRT-NPS, Duke University Medical Center, 2301 Erwin Rd, Durham, NC 27710. E-mail: andrew.g.miller@duke.edu.
}

DOI: $10.4187 /$ respcare.06626 


\section{RT-Driven Asthma Pathway in the PICU}

tors and systemic corticosteroids. ${ }^{4}$ Worldwide, approximately 500,000 children with status asthmaticus require admission to pediatric ICUs (PICUs) each year, with the number of PICU admissions increasing over time. ${ }^{5-8}$ Current data support the use of asthma pathways or protocols

\section{See the Related Editorial on Page 1445}

to reduce hospital length of stay (LOS) without increases in hospital readmissions. Patients admitted to the PICU were included in these studies, but outcomes for subjects in the PICU were not specifically evaluated. ${ }^{9-13}$ Brennan et $\mathrm{al}^{14}$ recently found that a de-escalation pathway was associated with modest decreases in PICU LOS, hospital LOS, and duration of continuous albuterol therapy.

At our institution, a pediatric asthma management clinical pathway is used for all patients admitted to Duke Children's Hospital, including those in the PICU. Prior to development of the asthma pathway, our mean LOS for all pediatric asthma patients was found to be longer than similar institutions. In particular, weaning the frequency of bronchodilators was noted to be a significant challenge in both the PICU and after PICU discharge. Thus, the pathway incorporated respiratory therapist (RT)-driven bronchodilator weaning and escalation using the Modified Pulmonary Index Score (MPIS), a validated asthma scoring system. ${ }^{9,15}$ The initial project was driven by our pediatric hospitalist service and general pediatric service with a focus on patients in the wards and in stepdown units. There was some concern from clinicians that the pathway was not appropriate for the PICU, so we needed to evaluate the efficacy of the pathway in the PICU setting.

This care pathway was previously reported to decrease hospital LOS for all pediatric asthma patients with no increase in readmission rates. ${ }^{9}$ The effect of the pathway on patients admitted to the PICU is unclear, as they were included in the original project but not examined as a specific subgroup. The purpose of this study was to examine the effect of our asthma pathway on outcomes of patients admitted to the PICU. We hypothesized that implementation of the asthma pathway would result in a statistically significant reduction in hospital LOS, PICU LOS, and duration of continuous albuterol therapy.

\section{Methods}

Subjects ages 2-17 y admitted to our PICU for asthma were identified using ICD-9 and ICD-10 codes. This project was exempt from institutional review board approval because it is a quality-improvement project. We chose to perform a before-and-after study instead of using qualityimprovement methodology because our quality-improvement efforts were not aimed at the PICU specifically. The

\section{QUICK LOOK}

\section{Current knowledge}

Asthma is a common reason for pediatric ICU (PICU) admission. Current data support the use of asthma pathways or protocols to reduce the length of hospital stay without increasing hospital readmissions. Previous studies have included PICU subjects but have not specifically evaluated their outcomes. There are limited data available evaluating the use of asthma pathways in the PICU.

\section{What this paper contributes to our knowledge}

For subjects admitted to our PICU, implementation of an asthma pathway that incorporated respiratory therapistdriven weaning of bronchodilators was associated with significant decreases in overall hospital length stay, PICU length of stay, and time on continuous albuterol.

original project examined our entire asthma population, but its efficacy in the PICU also required evaluation. Our PICU is a 16-bed tertiary care center, staffed around the clock by pediatric intensivists, critical care fellows, nurse practitioners, and pediatric RTs. Children requiring continuous albuterol therapy are required to be in either the PICU or the stepdown unit. The pre-pathway group included all subjects admitted to the PICU between July 2013 and June 2014. The post-pathway group included those admitted between July 2014 and November 2017. All subjects admitted to the PICU for asthma at any time during their hospital stay were included. Patients with cystic fibrosis, pneumonia, chronic lung disease, or those $<2$ y old were not eligible to be enrolled in the pathway. Exclusion criteria included home oxygen, home noninvasive ventilation (NIV), upper airway pathologies such as vocal cord dysfunction or subglottic stenosis, presence of tracheostomy, and invasive mechanical ventilation upon admission to the PICU.

Demographic data collected included age, weight, medical history, home medications, cause of exacerbation, route of admission, and respiratory support during admission. Medical history included prior ICU admission, NIV use, or intubation. Data were extracted via manual chart review and determined from admission history and physical or discharge summary for that hospital admission only. LOS data were calculated from hospital admission to hospital discharge. PICU LOS was calculated from PICU admission to the time when the patient left the PICU. Duration of continuous albuterol was calculated using the medication administration record and respiratory therapy documentation. Admission vitals and MPIS scores were the first documented in the PICU. 


\section{RT-Driven Asthma Pathway in the PICU}

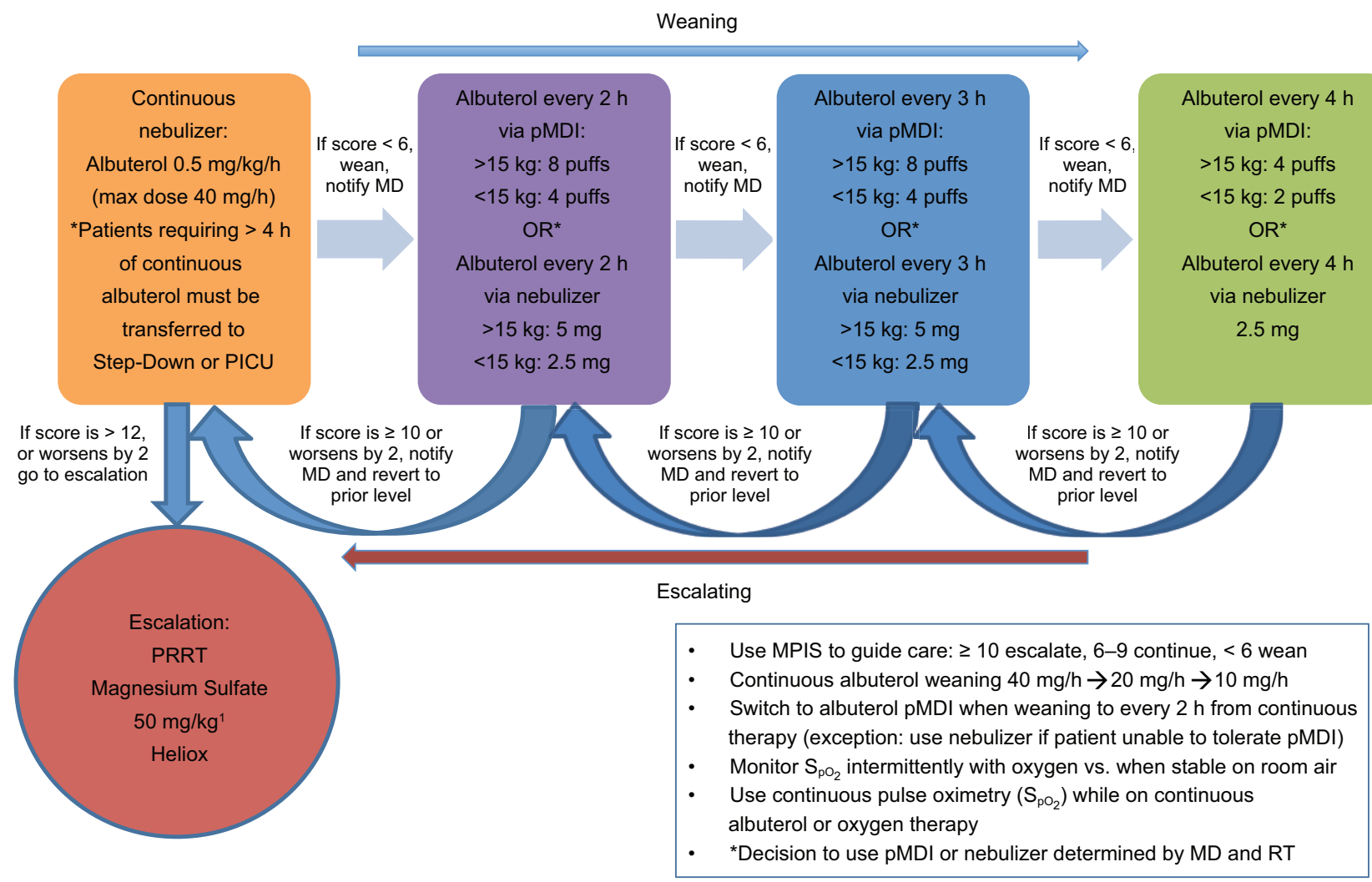

Fig. 1. Asthma treatment protocol at Duke Children's Hospital using the Modified Pulmonary Index Score (MPIS) to guide care. pMDI = pressurized metered-dose inhaler; MD = physician; RT = respiratory therapist; PRRT = pediatric rapid response team.

The pediatric asthma pathway was implemented in June 2014. The pathway uses an RT-driven bronchodilator frequency protocol guided by the MPIS (Fig. 1).15 The MPIS has been reported to have a high inter-rater reliability ( $\mathrm{R}$ values of 0.94 to 0.98 and excellent agreement on BlandAltman plots) between nurses, physicians, and RTs. ${ }^{15}$ The pathway directs RTs to independently increase (at MPIS $\geq 10$ ) or decrease (at MPIS $<6$ ) the frequency of bronchodilator therapy based on MPIS assessments performed every $2 \mathrm{~h}$ for those receiving continuous albuterol or at the scheduled frequency. Prior to pathway initiation, subjects were assessed a minimum of every $4 \mathrm{~h}$. Subjects with an MPIS $\geq 10$ receive continuous albuterol therapy at 20 $40 \mathrm{mg} / \mathrm{h}$. Once the MPIS is $<6$, continuous albuterol dosing is weaned or the patient is transitioned to treatments every $2 \mathrm{~h}$. Continuous albuterol was delivered via a Hope nebulizer (B\&B Medical Technologies, Carlsbad, CA) with aerosol mask or via syringe pump and vibrating mesh nebulizer for those on high-flow nasal cannula (HFNC) or NIV. HFNC prongs were sized to half the size of the subject's naris, and HFNC flow was titrated to meet subjects' inspiratory demand and $\mathrm{S}_{\mathrm{pO}_{2}}$ goal per our institution's clinical policy. All subjects received systemic corticosteroids every $6 \mathrm{~h}$. Heliox and intravenous magnesium sulfate administration were included as part of the path- way for subjects with MPIS $\geq 12$, and a single dose of intravenous magnesium is standard therapy for all patients requiring PICU admission. HFNC and NIV were not included as part of the pathway and were used as adjunct therapy at provider discretion. Data on intravenous magnesium, terbutaline, and aminophylline were collected. For magnesium, we were able to determine whether subjects received the drug but not whether they received it as a bolus for asthma or for electrolyte supplementation.

The hospital transitioned to a new electronic health record in June 2013; as a result, only 49 pre-pathway subjects were identified. Power analysis was subsequently performed using a 1:2.5 enrollment method to detect a $25 \%$ decrease in mean LOS with $80 \%$ power and an alpha of 0.05 . The pathway was implemented in June 2014. The post-pathway group was identified from an existing quality-improvement database that included all pediatric asthma in-patient admissions, and we enrolled all subjects who were eligible through November 2017. Data were extracted from the electronic medical record, entered into REDCap, and analyzed with SPSS v24 (SPSS, Chicago, Illinois). We utilized the chi-square test for categorical data, the unpaired $t$ test for normally distributed data, and the Mann-Whitney rank-sum test for non-normally distributed data. Propensity analysis was completed for sub- 
Table 1. Demographics

\begin{tabular}{|c|c|c|c|}
\hline & Pre-Pathway Group & Post-Pathway Group & $P$ \\
\hline Age, $y$, mean $\pm S D$ & $6.5 \pm 3.6$ & $7.4 \pm 3.7$ & .13 \\
\hline Gender $n$ ( $\%$ female $)$ & $17(35)$ & $67(44)$ & .28 \\
\hline Weight, kg, median (interquartile range) & $20.6(16.5-31.0)$ & $24.5(17.8-36.9)$ & .13 \\
\hline \multicolumn{4}{|l|}{ Home medications, $n(\%)$} \\
\hline Short-acting $\beta_{2}$ agonist & $44(90)$ & $131(85)$ & .40 \\
\hline ICS & $20(41)$ & $61(40)$ & .88 \\
\hline Long-acting $\beta_{2}$ agonist + ICS & $3(6)$ & $25(16)$ & .07 \\
\hline None & $3(6)$ & $21(14)$ & .16 \\
\hline \multicolumn{4}{|l|}{ History, $n(\%)$} \\
\hline Intubation & $1(2)$ & $4(3)$ & .83 \\
\hline ICU admission & $11(22)$ & $39(25)$ & .68 \\
\hline NIV & $0(0)$ & $7(5)$ & .13 \\
\hline None & $36(73)$ & $113(73)$ & .99 \\
\hline Cause of exacerbation, $n(\%)$ & & & .57 \\
\hline Viral & $26(53)$ & $94(61)$ & \\
\hline Exposure & $1(2)$ & $5(3)$ & \\
\hline Nonadherence, noncompliance & $2(4)$ & $10(6)$ & \\
\hline Other & $0(0)$ & $1(0.6)$ & \\
\hline Environmental & $2(4)$ & $8(5)$ & \\
\hline Unknown or undocumented & $18(37)$ & $36(23)$ & \\
\hline Route of admission to pediatric ICU, $n(\%)$ & & & .14 \\
\hline Emergency department & $30(61)$ & $66(43)$ & \\
\hline Hospital wards & $1(2)$ & $10(6)$ & \\
\hline Stepdown & $2(4)$ & $3(2)$ & \\
\hline Outside hospital & $15(31)$ & $72(47)$ & \\
\hline \multicolumn{4}{|c|}{$\begin{array}{l}\text { Pre-pathway group, } n=49 \text { subjects; post-pathway group, } n=154 \text { subjects. Data are presented as mean } \pm \mathrm{SD} \text {, median (interquartile range), or } n \text { (\%). } \\
\text { ICS }=\text { inhaled corticosteroid } \\
\text { NIV = noninvasive ventilation }\end{array}$} \\
\hline
\end{tabular}

jects receiving HFNC after observing an increase in HFNC use post-pathway. Subjects were matched based on age, weight, route of admission, home medications, and initial MPIS.

\section{Results}

We identified a total of 209 patients, with 203 subjects eligible to be included in our analysis; 6 patients were excluded for the following reasons: 2 for upper airway pathologies, 1 for invasive ventilation, 2 for home NIV, and 2 for home oxygen. One subject was excluded for both home NIV and home oxygen. The pre-pathway group included 49 subjects, and the post-pathway group included 154. There were no differences for age, weight, gender, home medications, cause of exacerbation, medical history, or route of admission between groups (Table 1). Median hospital LOS is included as a run chart (Figure 2).

There were significant decreases between the pre- and post-pathway groups in median (interquartile range [IQR]) hospital LOS (4.4 [2.9-6.6] versus $2.7[1.6-4.0] \mathrm{d}$, $P<.001)$, median PICU LOS (2.1 [1.3-4.0] versus 1.6
[0.8-2.4] d, $P=.003)$, and median time on continuous albuterol 39 [25-85] versus 27 [13-42] h, $P=.001$ ). There was no significant difference between groups for PICU readmissions ( $0 \%$ vs $0.6 \%, P=.57$ ). Significantly more subjects in the post-pathway group were placed on HFNC $(32 \%$ vs $6 \%, P=.001)$ or NIV $(10 \%$ vs $4 \%$, $P=.02)$. Subjects in the post-pathway group had lower admission heart rate $(152 \pm 19$ vs $160 \pm 18$ beats/min, $P=.006)$ and breathing frequency $(39 \pm 13$ vs $45 \pm 12$ breaths $/ \mathrm{min}, P=.006$ ). There were no statistically significant differences for $\mathrm{S}_{\mathrm{pO}_{2}}, \mathrm{~F}_{\mathrm{IO}_{2}}$, temperature, or intravenous medication use (Table 2). There were no statistically significant differences in heart rate, breathing frequency, $\mathrm{S}_{\mathrm{pO}_{2}}$, or $\mathrm{F}_{\mathrm{IO}_{2}}$ between groups at the time when continuous albuterol was discontinued.

Given the noted increase in the use of HFNC in the post-intervention cohort, a propensity score matching analysis was completed for subjects receiving HFNC. For this analysis, 91 pathway subjects were matched based on age, weight, route of admission, home medications, and initial MPIS. Subjects receiving HFNC had a nonsignificant increase in their median (IQR) hospital LOS, when com- 


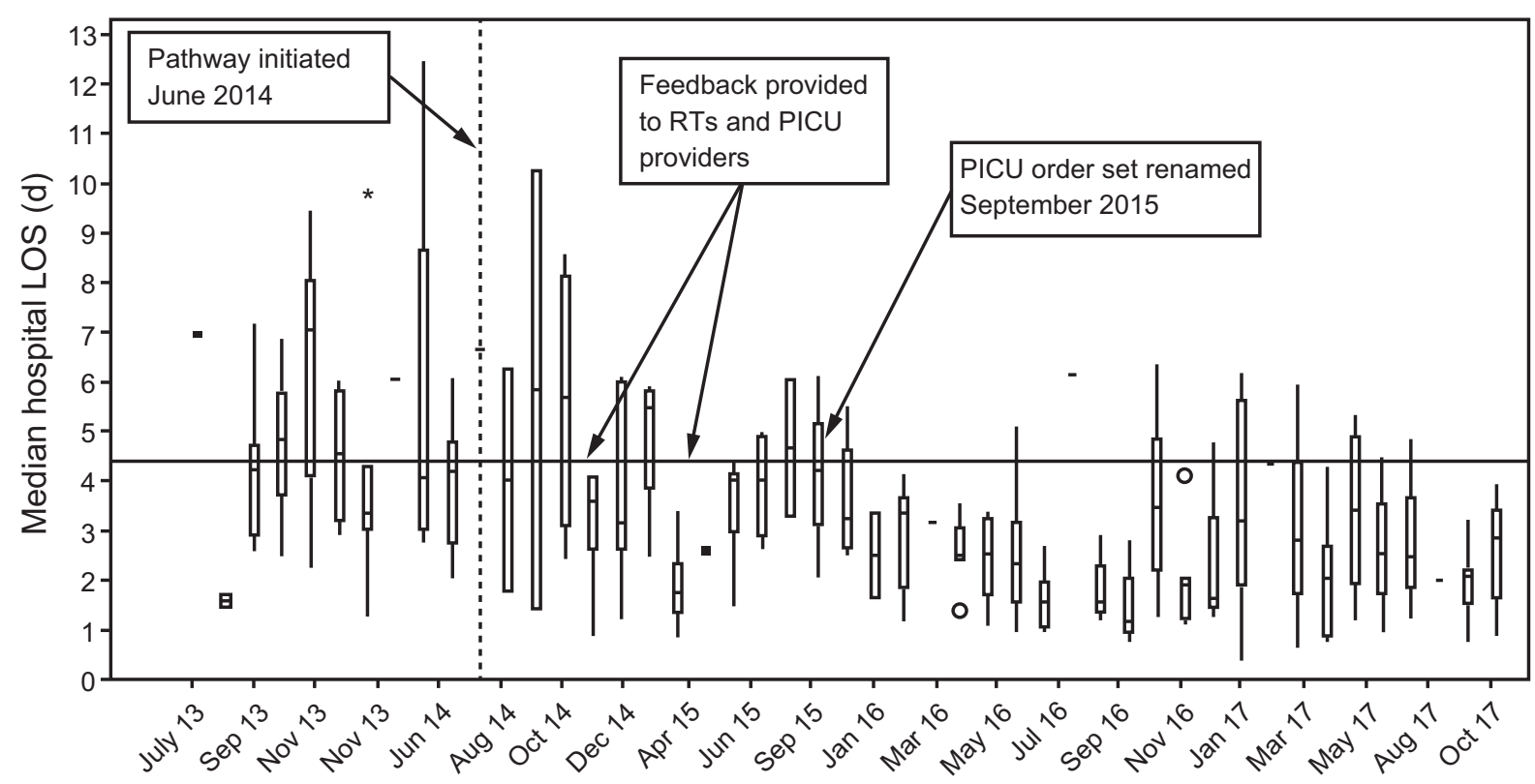

Fig. 2. Median hospital length of stay (LOS). RT = respiratory therapist; PICU = pediatric ICU.

pared to subjects on aerosol mask (3.42 [2.41-4.85] vs 2.79 [2.04-3.54] $P=.17)$. Median PICU LOS was 1.93 (IQR 1.18-3.34) versus 1.62 (IQR 1.05-2.26) d $(P=.17)$. There were no differences found for age, weight, gender, inhaled corticosteroid use, MPIS, or route of admission between groups. More subjects in the HFNC group $(26 \%$ vs $9 \%, P=.04$ ) took a long-acting $\beta$ agonist and inhaled corticosteroid at home. Results are summarized in Table 3.

\section{Discussion}

For subjects admitted to our PICU with status asthmaticus, implementation of an asthma pathway that incorporated RT-driven weaning of bronchodilators was associated with significant decreases in overall hospital LOS, PICU LOS, and duration of continuous albuterol. These data represent a subset of a larger quality-improvement report from our institution that evaluated the impact of the asthma pathway on all children hospitalized with asthma exacerbations and found a decrease in hospital LOS of 0.6 d. ${ }^{9}$ In our study, we found that the subset of subjects admitted to the PICU for status asthmaticus spent a median of 12 fewer hours in the PICU but were discharged home a median of $1.7 \mathrm{~d}$ sooner. Thus, the improvement in overall hospital LOS in our study was in large part due to decreased time (median of $1.2 \mathrm{~d}$ ) on the wards after being discharged from the PICU. Utilizing the pathway to decrease frequency of bronchodilator treatment and providing clear guidance for discharge criteria likely identified subjects ready to be discharged sooner. Heart rate and breathing frequency were lower in the post-pathway group, suggesting potentially less severe exacerbations; however, subjects were also nonsignificantly older, larger, and there were no differences in adjunctive medication use between groups. Without a validated scoring system (eg, MPIS) for the pre-protocol cohort, it was not possible to adequately evaluate disease severity between the 2 groups.

Our institution uses the same pathway throughout all the in-patient pediatric areas, and it is likely that our success was related to a high level of RT and provider buy-in. There was some resistance initially from clinicians in the PICU, largely concerned about elevated heart rates related to continuous albuterol resulting in an MPIS above the weaning criteria. RTs at our institution are highly autonomous, managing mechanical ventilation via institutional protocol, and are thus experienced in using pathways to manage patients. Feedback and training were provided during the initial rollout as well as periodically since the pathway was initiated. ${ }^{9}$ The use of the MPIS helped provide a consistent assessment of each subject's status and allowed early detection of subjects who were not responding to therapy and required escalation in therapy. Prior to pathway initiation, individual patient management was dependent on physician assessment and order entry. Weaning was often based on subjective criteria such as breath sounds instead of a validated asthma-scoring system or objective measures of air-flow obstruction. As part of the pathway, subjects receiving continuous albuterol therapy were assessed every $2 \mathrm{~h}$ instead of every $4 \mathrm{~h}$ as was the practice prior to pathway introduction. The frequent respi- 


\section{RT-Driven Asthma Pathway in the PICU}

Table 2. Subject Outcomes

\begin{tabular}{|c|c|c|c|}
\hline & Pre-Pathway Group & Post-Pathway Group & $P$ \\
\hline Hospital LOS, d & $4.4(2.9-6.6)$ & $2.7(1.6-4.0)$ & $<.001$ \\
\hline Pediatric ICU LOS, d & $2.1(1.3-4.0)$ & $1.6(0.8-2.4)$ & .003 \\
\hline Readmission to pediatric ICU, $n(\%)$ & $0(0)$ & $1(0.6)$ & .57 \\
\hline Discharged directly from pediatric ICU, $n(\%)$ & $9(18)$ & $37(24)$ & .34 \\
\hline Received continuous albuterol, $n(\%)$ & $44(90)$ & $124(81)$ & .13 \\
\hline Duration of continuous albuterol, $\mathrm{h} *$ & $39(25-85)$ & $27(13-42)$ & .001 \\
\hline \multicolumn{4}{|l|}{ Respiratory support during admission, $n(\%)$} \\
\hline High-flow nasal cannula & $3(6)$ & $50(32)$ & .001 \\
\hline Noninvasive ventilation & $3(6)$ & $16(10)$ & .02 \\
\hline Heliox & $23(47)$ & $46(30)$ & .053 \\
\hline Intubated & $0(0)$ & $2(1)$ & .52 \\
\hline \multicolumn{4}{|l|}{ Admission data } \\
\hline Heart rate, beats/min & $160 \pm 18$ & $152 \pm 19$ & .006 \\
\hline Frequency, breaths/min & $45 \pm 12$ & $39 \pm 13$ & .006 \\
\hline Temperature, ${ }^{\circ} \mathrm{C}$ & $37 \pm 0.45$ & $37 \pm 0.49$ & .56 \\
\hline $\mathrm{S}_{\mathrm{pO}_{2}}$ & $95 \pm 4.3$ & $96 \pm 3.0$ & .16 \\
\hline $\mathrm{F}_{\mathrm{IO}_{2}}$ & $0.36 \pm 0.24$ & $0.42 \pm 0.28$ & .15 \\
\hline MPIS & N/A & $9(7-12)$ & N/A \\
\hline \multicolumn{4}{|l|}{ When continuous albuterol discontinued } \\
\hline Heart rate, beats/min & $134 \pm 22$ & $135 \pm 22$ & .75 \\
\hline Frequency, breaths/min & $37 \pm 11$ & $34 \pm 10$ & .054 \\
\hline Temperature, ${ }^{\circ} \mathrm{C}$ & $37 \pm 0.15$ & $37 \pm 0.24$ & .57 \\
\hline $\mathrm{S}_{\mathrm{pO}_{2}}$ & $96 \pm 2.2$ & $97 \pm 2.4$ & .39 \\
\hline $\mathrm{F}_{\mathrm{IO}_{2}}$ & $0.24 \pm 0.12$ & $0.26 \pm 0.16$ & .37 \\
\hline MPIS & N/A & $5(4-6)$ & N/A \\
\hline $\begin{array}{l}\text { Pre-pathway group, } n=49 \text { subjects; post-pathway group, } n= \\
* n=44 \text { of } 124 \text { subjects. } \\
\text { LOS }=\text { length of stay } \\
\text { MPIS = Modified Pulmonary Index Score } \\
\text { N/A = not applicable }\end{array}$ & presented as mean $\pm \mathrm{SD}$, me & ge), or $n(\%)$ & \\
\hline
\end{tabular}

Table 3. Propensity Score Matched Cohort

\begin{tabular}{|c|c|c|c|}
\hline & HFNC & Aerosol Mask & $P$ \\
\hline Age, y & $7(4-10)$ & $6(5-8)$ & .70 \\
\hline Weight, kg & $25.4(16.8-43.0)$ & $21.7(17.2-28.2)$ & .24 \\
\hline Gender, $n$ ( $\%$ male $)$ & $24(51)$ & $28(64)$ & .23 \\
\hline $\mathrm{LABA}+\mathrm{ICS}, n(\%)$ & $12(26)$ & $4(9)$ & .040 \\
\hline $\mathrm{ICS}, n(\%)$ & $16(34)$ & $21(48)$ & .18 \\
\hline MPIS & $11(9-13)$ & $12(8-13)$ & .97 \\
\hline \multicolumn{4}{|l|}{ Admission route, $n(\%)$} \\
\hline Outside hospital & $24(55)$ & $33(75)$ & .13 \\
\hline Emergency department & $17(39)$ & $9(20)$ & \\
\hline Hospital wards & $3(7)$ & $2(5)$ & \\
\hline Hospital LOS, d & $3.42(2.41-4.85)$ & $2.79(2.04-3.54)$ & .17 \\
\hline Pediatric ICU LOS, d & $1.93(1.18-3.34)$ & $1.62(1.05-2.26)$ & .17 \\
\hline $\begin{array}{l}\text { Duration of continuous } \\
\text { albuterol, } \mathrm{h}\end{array}$ & $29(18-60)$ & $28(15.5-48)$ & .89 \\
\hline \multicolumn{4}{|c|}{$\begin{array}{l}\text { HFNC group, } n=44 \text { subjects; aerosol mask group, } n=47 \text { subjects. Data are presented as } \\
\text { median (interquartile range) or } n(\%) . \\
\text { LABA = long-acting } \beta \text { agonist } \\
\text { ICS = inhaled corticosteroid } \\
\text { MPIS = Modified Pulmonary Index Score } \\
\text { LOS = length of stay }\end{array}$} \\
\hline
\end{tabular}

ratory assessments likely identified patients eligible to be weaned sooner, and the RTs' ability to immediately act on that assessment without a separate order expedited the weaning process. Although the increased frequency of assessment increased RT work load, the RTs were able to incorporate the pathway into daily practice without an increase in the number of RTs staffing the PICU, likely due to the high level of buy-in. Use of RT resources was not measured as part of our project; however, this is an important consideration for future projects.

There are limited data to date evaluating the use of asthma pathways in the PICU environment. Brennan et al ${ }^{14}$ evaluated a de-escalation pathway in a quality-improvement project designed to decrease variability in PICU LOS in a tertiary PICU. Their pathway reduced median PICU LOS by $3 \mathrm{~h}$, duration of continuous albuterol by a median of $3.5 \mathrm{~h}$, and hospital LOS by $6 \mathrm{~h}$. With our pathway, we observed a larger impact, with the median PICU LOS decreasing by $0.5 \mathrm{~d}(12 \mathrm{~h})$, duration of continuous albuterol by $10 \mathrm{~h}$, and median overall hospital LOS by $1.7 \mathrm{~d}$. Their study did not include vital sign data or other thera- 


\section{RT-Driven Asthma Pathway in the PICU}

pies delivered, so it is challenging to compare our results. Our baseline LOS was longer than they reported, thus we may have had a larger opportunity for improvement. Notably, our primary outcome was total hospital LOS, whereas they focused on reducing variability in clinical practice. Other studies have enrolled subjects outside the PICU, ${ }^{10,12}$ only included $9 \%$ of subjects admitted to PICU, ${ }^{11}$ or did not report the proportion of subjects admitted to PICU. ${ }^{13}$

After the implementation of this pathway, we noted increases in HFNC and NIV use; the impact of these adjunctive therapies on our outcome measures is unclear. We have observed clinically an increased tendency to place patients on HFNC immediately upon arrival in our PICU, both for disease severity and for patient comfort. We were unable to determine retrospectively whether subjects were placed on HFNC due to mask intolerance or respiratory distress, but, given that HFNC and NIV use were higher post-protocol despite lower heart rates and breathing frequencies, this likely reflects clinician bias toward early initiation of HFNC. Our propensity analysis revealed that HFNC was associated with a non-statistically significant longer LOS when compared to aerosol face mask. Thus, an increase in HFNC use post pathway implementation is unlikely to explain decreases in PICU and hospital LOS. To date, no randomized, controlled trials of HFNC or NIV in pediatric asthma have been performed in the PICU. The effects of HFNC on aerosol delivery and escalation of therapy remains controversial. ${ }^{16-22}$

The role of NIV in pediatric asthma is also unclear due to lack of data from high-quality randomized, controlled trials. Published data indicate that it is safe, it decreases respiratory distress, and it is associated with lower rates of endotracheal intubation. ${ }^{23-26}$ The small increase in NIV use in the postpathway group may have been due to improved identification of those failing to respond to other therapies, but we were unable to capture specific reasons why subjects were placed on NIV. It is likely that the difference was due to chance and does not represent a true change in practice as we have used NIV frequently in asthma for many years prior to development of the pathway.

\section{Limitations}

This study has several limitations. As a retrospective review, we were limited to data available in the medical record, including a limited pre-pathway sample size. Factors other than the pathway may have contributed to the results. In particular, an increase in HFNC and NIV use may be significant confounders, despite the propensity analysis suggesting otherwise. The admission heart rates and breathing frequencies were statistically significantly lower in the post-intervention group, which may have indicated that their asthma exacerbations were less severe. However, the absolute differences in these vital signs between the
2 groups were small, and baseline saturations and oxygen requirements were similar. We did not measure costs or the effect on RT work load in the PICU. PICU LOS is heavily influenced by bed availability, and many of our subjects were discharged directly from the PICU. Finally, there are no standardized PICU discharge criteria for patients with asthma.

\section{Conclusion}

The implementation of an RT-driven asthma pathway was associated with decreased hospital LOS, PICU LOS, and time on continuous albuterol for patients admitted to the PICU with status asthmaticus.

\section{REFERENCES}

1. Akinbami LJ, Moorman JE, Bailey C, Zahran HS, King M, Johnson $\mathrm{CA}$, et al. Trends in asthma prevalence, health care use, and mortality in the United States, 2001-2010. NCHS Data Brief 2012(94):1-8.

2. Jones BP, Paul A. Management of acute asthma in the pediatric patient: an evidence-based review. Pediatr Emerg Med Pract 2013; 10(5):1-23.

3. Bourgeois FT, Monuteaux MC, Stack AM, Neuman MI. Variation in emergency department admission rates in US children's hospitals. Pediatrics 2014;134(3):539-545.

4. Rehder KJ. Adjunct therapies for refractory status asthmaticus in children. Respir Care 2017;62(6):849-865.

5. Nievas IF, Anand KJ. Severe acute asthma exacerbation in children: a stepwise approach for escalating therapy in a pediatric intensive care unit. J Pediatr Pharmacol Ther 2013;18(2):88-104.

6. Al-Eyadhy AA, Temsah MH, Alhaboob AA, Aldubayan AK, Almousa NA, Alsharidah AM, et al. Asthma changes at a pediatric intensive care unit after 10 years: observational study. Ann Thorac Med 2015;10(4):243-248.

7. Hartman ME, Linde-Zwirble WT, Angus DC, Watson RS. Trends in admissions for pediatric status asthmaticus in New Jersey over a 15-year period. Pediatrics 2010;126(4):e904-911.

8. Strid JM, Gammelager H, Johansen MB, Tonnesen E, Christiansen CF. Hospitalization rate and 30-day mortality among patients with status asthmaticus in Denmark: a 16-year nationwide populationbased cohort study. Clin Epidemiol 2013;5:345-355.

9. Bartlett KW, Parente VM, Morales V, Hauser J, McLean HS. Improving the efficiency of care for pediatric patients hospitalized with asthma. Hosp Pediatr 2017;7(1):31-38.

10. Johnson KB, Blaisdell CJ, Walker A, Eggleston P. Effectiveness of a clinical pathway for inpatient asthma management. Pediatrics 2000; 106(5):1006-1012.

11. Kelly CS, Andersen CL, Pestian JP, Wenger AD, Finch AB, Strope GL, et al. Improved outcomes for hospitalized asthmatic children using a clinical pathway. Ann Allergy Asthma Immunol 2000;84(5):509-516.

12. McDowell KM, Chatburn RL, Myers TR, O'Riordan MA, Kercsmar CM. A cost-saving algorithm for children hospitalized for status asthmaticus. Arch Pediatr Adolesc Med 1998;152(10):977-984.

13. Wazeka A, Valacer DJ, Cooper M, Caplan DW, DiMaio M. Impact of a pediatric asthma clinical pathway on hospital cost and length of stay. Pediatr Pulmonol 2001;32(3):211-216.

14. Brennan S, Lowrie L, Wooldridge J. Effects of a PICU status asthmaticus de-escalation pathway on length of stay and albuterol use. Pediatr Crit Care Med 2018;19(7):658-664. 


\section{RT-Driven Asthma Pathway in the PICU}

15. Carroll CL, Sekaran AK, Lerer TJ, Schramm CM. A modified pulmonary index score with predictive value for pediatric asthma exacerbations. Ann Allergy Asthma Immunol 2005;94(3):355-359.

16. Ballestero Y, De Pedro J, Portillo N, Martinez-Mugica O, AranaArri E, Benito J. Pilot clinical trial of high-flow oxygen therapy in children with asthma in the emergency service. J Pediatr 2018;194: 204-210 e203.

17. Pilar J, Modesto IAV, Lopez-Fernandez YM, Lopez-Macias O, Garcia-Urabayen D, Amores-Hernandez I. High-flow nasal cannula therapy versus non-invasive ventilation in children with severe acute asthma exacerbation: an observational cohort study. Med Intensiva 2017;41(7):418-242.

18. DiBlasi RM. Clinical controversies in aerosol therapy for infants and children. Respir Care 2015;60(6):894-914.

19. Ari A, Harwood R, Sheard M, Dailey P, Fink JB. In vitro comparison of heliox and oxygen in aerosol delivery using pediatric high flow nasal cannula. Pediatr Pulmonol 2011;46(8):795-801.

20. Dailey PA, Harwood R, Walsh K, Fink JB, Thayer T, Gagnon G, et al. Aerosol delivery through adult high flow nasal cannula with heliox and oxygen. Respir Care 2017;62(9):1186-1192.
21. Carroll CL. Heliox for children with acute asthma: has the sun set on this therapy? Pediatr Crit Care Med 2010;11(3):428-429.

22. Rodrigo GJ, Castro-Rodriguez JA. Heliox-driven beta2-agonists nebulization for children and adults with acute asthma: a systematic review with meta-analysis. Ann Allergy Asthma Immunol 2014;112(1):29-34.

23. Basnet S, Mander G, Andoh J, Klaska H, Verhulst S, Koirala J. Safety, efficacy, and tolerability of early initiation of noninvasive positive pressure ventilation in pediatric patients admitted with status asthmaticus: a pilot study. Pediatr Crit Care Med 2012;13(4):393-398.

24. Beers SL, Abramo TJ, Bracken A, Wiebe RA. Bilevel positive airway pressure in the treatment of status asthmaticus in pediatrics. Am J Emerg Med 2007;25(1):6-9.

25. Mayordomo-Colunga J, Medina A, Rey C, Concha A, Menendez S, Arcos ML, et al. Non-invasive ventilation in pediatric status asthmaticus: a prospective observational study. Pediatr Pulmonol 2011; 46(10):949-955.

26. Williams AM, Abramo TJ, Shah MV, Miller RA, Burney-Jones C, Rooks S, et al. Safety and clinical findings of BiPAP utilization in children $20 \mathrm{~kg}$ or less for asthma exacerbations. Intensive Care Med 2011;37(8):1338-1343

This article is approved for Continuing Respiratory Care Education credit. For information and to obtain your CRCE

(free to AARC members) visit

www.rcjournal.com

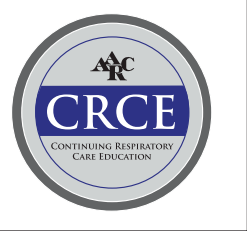

